\title{
PROGRESS IN SUPERCONDUCTING QUBITS
}

\author{
Preparation and Measurement of Three-Qubit Entanglement \\ in a Superconducting Circuit. \\ L. DiCarlo, M. D. Reed, L. Sun, B. R. Johnson, J. M. Chow, J. M. Gambetta, L. Frunzio, \\ S. M. Girvin, M. H. Devoret, R. J. Schoelkopf \\ arXiv:1004.4324
}

\section{Generation of Three-Qubit Entangled States using Superconducting Phase Qubits.}

M. Neeley, R. C. Bialczak, M. Lenander, E. Lucero, M. Mariantoni, A. D. OĆonnell, D. Sank, H. Wang, M. Weides, J. Wenner, Y. Yin, T. Yamamoto, A. N. Cleland, J. M. Martinis arXiv:1004.4246

Quantum Non-demolition Detection of Single Microwave Photons in a Circuit. B. R. Johnson, M. D. Reed, A. A. Houck, D. I. Schuster, Lev S. Bishop, E. Ginossar, J. M. Gambetta, L. DiCarlo, L. Frunzio, S. M. Girvin, R. J. Schoelkopf arXiv:1003.2734

Quantum process tomography of a universal entangling gate implemented with Josephson phase qubits.

R. C. Bialczak, M. Ansmann, M. Hofheinz, E. Lucero, M. Neeley, A. D. O?́Connell, D. Sank, H. Wang, J. Wenner, M. Steffen, A. N. Cleland, J. M. Martinis arXiv:0910.1118; Nature Physics 6, 409 - 413 (2010)

\section{Recommended with a Commentary by David DiVincenzo, IBM T. J. Watson Research Center}

The tremendous progress on qubits and quantum logic circuits made recently with superconducting circuits, as indicated by the four papers above, arises largely from three simple facts: 
1. An on-chip superconducting transmission line is a superb medium for guided radiation. If we could make the chip, and the cryostat, long enough, a single microwave photon could be transmitted many kilometers without loss, as far as infrared photons travel in a good optical fiber.

2. Open-cicuit ends of these transmission lines are perfect mirrors, more than sufficient to make a cavity resonator that holds photons for their full c. 10 microsecond lifetime.

3. By incorporating superconducting tunnel junctions into such resonators, they can be made nonlinear enough to exhibit a photon blockade: the dynamics of the resonator can be confined to the subspace of $|0\rangle$ and $|1\rangle$. Thus is the qubit made.

Previous work, armed with these facts, has made qubits of ever-improving coherence and reliability; made coupled qubit-resonator systems that accurately embody the physics of atoms strongly coupled to the quantum electrodynamic field of a cavity; and has mediated coupling of two qubits via such a cavity, achieving the controlled creation of entanglement that is the essence of the quantum logic gate.

The current activity builds on these results in the two interrelated directions of precision and complexity. Functioning three and four qubit circuits are achieved in the papers of DiCarlo et al and Neeley et al., and desired multi-qubit entangled states are achieved. A circuit with two resonators is used in Johnson et al. to attain a new level of capability in the quantum non-demolition measurement of the number of photons in a resonator, with a repeatability of $90 \%$. Bialczak et al. show the precise and versatile implementation of two-qubit gates, with high on-off ratio (300), and a precise characterization of the fidelity of the achieved gate operations (c. 65\%).

These papers all make valiant efforts to convince us that there is some fundamental new physics achieved in these advancements in complexity and precision. We get a realization of entanglement witnesses, a precise test of the Mermin-Bell inequalities, and the violation of biseparability, on the GHZ states (Greenberger, Horne, and Zeilinger), $(|G H Z\rangle=|000\rangle+|111\rangle)$ and the $\mathrm{W}$ states (just $\mathrm{W} ;|W\rangle=|001\rangle+|010\rangle+|100\rangle$ ). One might imagine that this stirs up a veritable hornet's nest of philosophers and the like, fresh from the Bell inequality wars. But there's not a great deal of this, really; I don't think much of anybody gives a hoot about the detector loopholes, or the speed-of-light loopholes, in these experiments. For my own 
taste, I am actually somewhat more interested in the much-ridiculed factoring of fifteen as a milestone for the next round of experiments.

The experiments are breathtaking nonetheless. But there is a basic conundrum for the physicist in this field: as these experiments grow to the compexity (tens or hundreds of qubits) and precision (99\% + fidelities) needed to have a fundamental influence on computer science, they will long since cease to be "fundamental" as physics experiments. Not every addition of a qubit, or of another nine of fidelity, will be a celebrated paper in a famous physics magazine. I hope this fact won't quench the physicists' enthusiasm for the work. It shouldn't. Perhaps their model should be metrology: sometimes (or, perhaps, inevitably), the painstaking, unglamorous addition of nines to the precision of an experiment has been the vehicle to basic new achievements and discoveries.

As a point in this direction, I observe one thing about these experiments which is not well featured by the papers themselves: as a purely technical matter, the experiments (and new ones in the pipeline) confidently send more and more microwave energy onto their chips. Virtually all of this energy flows right out of the chip again, with almost none of it (less and less, in fact, as the devices improve) dissipated as heat on the chip. Actually, the generated heat is a minuscule fraction of the $k T \ln 2$ dissipation per boolean logic operation as given by the naive application of Landauer's principle. So, these experiments are already, in a small way, a realization of nearly dissipationless, "reversible" computing on an integrated circuit. This alone could be a decisive factor in the future development of this work. 